Servicio de Medicina Interna Hospital San José, Santiago, Chile. Becaria Medicina Interna, Universidad de Santiago de Chile.

Recibido el 3 de agosto de 2012, aceptado el 2 de octubre de 2012

Correspondencia a: Dra. Anne-Marie

Chassin-Trubert Contreras Cirujano Guzmán 70 departamento 1303 Providencia, Santiago. E-mail: annechassin@ gmail.com

\section{Disección carotídea en paciente con síndrome de Ehlers-Danlos tipo vascular}

\author{
ANNE-MARIE CHASSIN-TRUBERT CONTRERAS
}

\section{Carotid dissection associated with Ehlers-Danlos syndrome. Report of one case}

In the vascular type of Ehlers-Danlos syndrome there is a defect in the synthesis of collagen type III, important constituent of the blood vessel wall, which may cause arterial rupture and dissection. We report a 15-year-old girl with a history of EhlersDanlos syndrome, who suffered an ischemic stroke secondary to dissection of the right internal carotid artery. She was managed conservatively with antiplatelet agents with a good clinical response.

(Rev Med Chile 2013; 141: 392-395).

Key words: Carotid artery, internal, dissection; Ehlers-Danlos syndrome; Stroke.
$\mathrm{E}$ 1 síndrome de Ehlers-Danlos (SED) es una enfermedad hereditaria asociada a mutación del gen del colágeno, dando origen a distintos subtipos según el gen afectado ${ }^{1}$. La mutación más frecuente es las de los genes COL5A1 y COL5A2 que codifican para el colágeno tipo $\mathrm{V}^{2}$. La prevalencia estimada varía entre $1 / 10.000$ y $1 / 25.000$ habitantes $^{3}$. Según la clasificación de Villefranche, se divide en seis subtipos: tipo clásico, tipo hiperlaxitud, tipo vascular, tipo cifoescoliosis, tipo artrocalasia y tipo dermatosparaxis ${ }^{4}$, siendo el tipo hiperlaxitud el más frecuente $(80 \%$ de los (asos) $)^{5}$. El SED tipo vascular, anteriormente conocido como SED tipo IV, es la forma más grave de presentación. Se hereda como rasgo autosómico dominante e involucra deficiencia del colágeno tipo III por mutación del gen COL3A1 cuyo locus se encuentra en el brazo largo del cromosoma 2, en la posición $2 \mathrm{q} 24.3-\mathrm{q} 31^{6-8}$. Representa 5\%-10\% del total de casos de SED, con una prevalencia estimada de 1-2/100.000 habitantes. Clínicamente destacan rasgos faciales característicos, como la acrogeria con labios finos, piel delgada, orejas sin lóbulos, nariz puntiaguda, mejillas hundidas y prominentes ojos fijos, además se caracteriza por piel translúcida con vasos subcutáneos visibles, equimosis y hematomas fáciles ${ }^{9}$. Es la forma más peligrosa de SED, ya que presenta graves complicaciones arteriales, digestivas y uterinas. Se afectan principalmente las arterias de diámetro grande y medio $^{10}$. El mayor compromiso vascular es de la aorta torácica y son habituales las disecciones de las arterias vertebrales y carótidas en sus segmentos extra e intracraneal. Los eventos vasculares se presentan habitualmente entre la tercera y cuarta década de la vida. El diagnóstico definitivo se realiza mediante estudio genético o biopsia de piel para cultivo de fibroblastos. No existe un tratamiento específico y se estima que $25 \%$ de los pacientes tendrá una complicación antes de los 25 años y más de $80 \%$ a los 40 años.

Se presenta un caso poco frecuente de síndrome de Ehlers-Danlos tipo vascular asociado a disección carotidea y se discute la conducta terapéutica.

\section{Caso clínico}

Mujer de 15 años de edad, chilena, con antecedente de síndrome de Ehlers-Danlos tipo vascular diagnosticado mediante estudio genético a los 8 años de edad; consultó por presentar cuadro de 5 días de evolución caracterizado por cefalea intermitente holocránea de intensidad 8/10, debilidad generalizada y parestesias de extremidad superior izquierda, estableciéndose posteriormente una hemiparesia facio braquio crural izquierda asociada a 
disartria. No presentó compromiso de conciencia ni convulsiones. Refirió que los síntomas aparecieron de forma espontánea, sin causa aparente.

Examen físico general: piel fina y translúcida con múltiples equímosis de predominio en miembros superiores. Examen neurológico: vigil, orientada temporo-espacialmente, campo visual normal, afasia de expresión, desviación de comisura labial hacia derecha, hemiparesia faciobraquiocrural izquierda con motilidad M2. Reflejo plantar con extensión a izquierda. Sin déficit sensitivo. Sin signos meníngeos.

Exámenes de laboratorio: parámetros dentro de rango normal. La tomografía axial computarizada (TAC) cerebral sin contraste evidenció un infarto subcortical derecho y se hospitalizó para estudio y tratamiento, iniciándose terapia neuroprotectora. Al segundo día, se realizó TAC cerebral con contraste que mostró un infarto subagudo silviano derecho que comprometía territorios profundo y superficial, además de una pequeña hemorragia intracraneal capsular posterior- corona radiada derecha de $9 \mathrm{~mm}$ de diámetro.

Debido al antecedente de síndrome de EhlersDanlos tipo IV, se decidió completar estudio con resonancia nuclear magnética de encéfalo complementado con angioresonancia magnética con contraste y angioresonancia de vasos del cuello, en la que destacó un infarto agudo-subagudo que comprometía los territorios de la arteria cerebral anterior y cerebral media, comprometiendo cabeza de caudado, núcleo lentiforme, región frontoinsular a derecha, así como también a nivel giral parietal anterior ipsilateral. Sin evidencias de transformación hemorrágica ni efecto de masa (Figura 1).

En estudio de polígono, destacó la falta de representación de la arteria carótida interna derecha que como se demostró en el estudio de vasos del cuello presentaba una disección próxima a su origen (Figura 2). Las arterias cerebral anterior y cerebral media derechas se reconstituyeron a través de la comunicante anterior. En estudio de perfusión, se evidenció significativo mistmatch. Como variante de arco aórtico la arteria subclavia izquierda nacía distal a la subclavia derecha (subclavia aberrante). Resto de estudio sin hallazgos significativos.

Considerando la presencia de mismatch, donde el tejido de riesgo era mayor al infartado, se decidió que la acción más importante era mejorar la

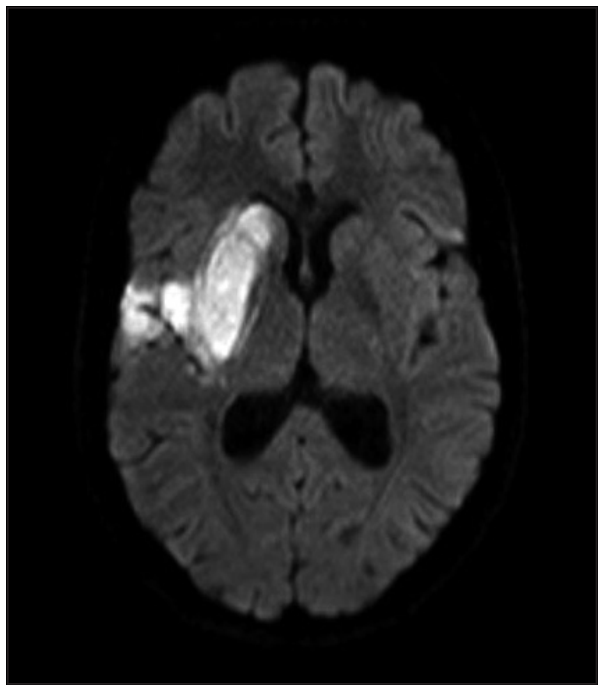

Figura 1. Resonancia nuclear magnética de encéfalo: compromiso isquémico de territorios de la arteria cerebral anterior y la arteria cerebral media, comprometiendo cabeza de caudado, núcleo lentiforme, región frontoinsular a derecha, así como también a nivel giral parietal anterior ipsilateral.

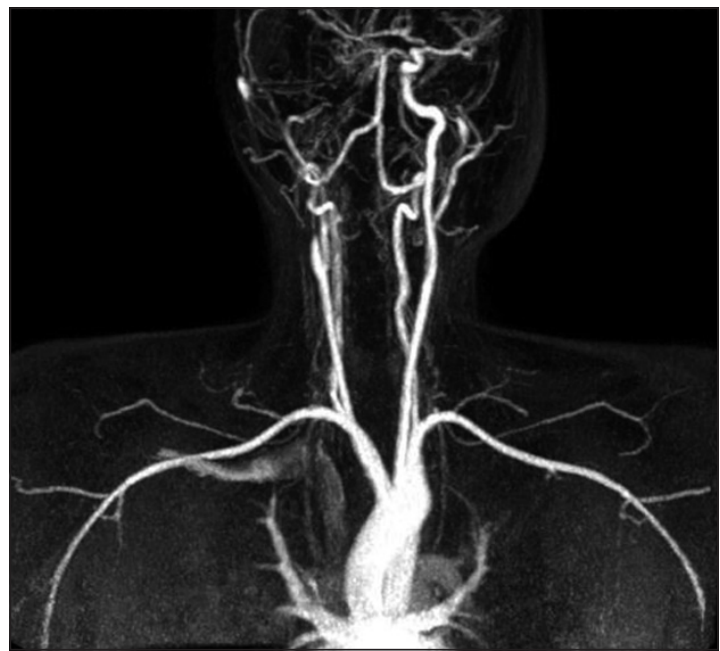

Figura 2. Angioresonancia de vasos del cuello: como variante de arco aórtico, la arteria subclavia izquierda nace distal a la subclavia derecha (subclavia aberrante).

presión arterial media (PAM) y mantenerla entre $80-90 \mathrm{mmHg}$ de manera de optimizar el flujo colateral, apoyándose con drogas vasoactivas los primeros 3 días. Presentó un episodio de desconexión asociado a relajación de esfínteres, por lo que se solicitó un electroencefalograma que resultó alterado por presencia de lentitud theta delta 
intermitente fronto centro temporal derecha que se acentuaba durante la hiperventilación (vigilia, somnolencia, hiperventilación, foto estimulación, 16-32 canales). Equipo de neurología descartó crisis de ausencia y planteó que ante un nuevo episodio convulsivo se debía iniciar terapia anticonvulsivante con lamotrigina, bajo sospecha de crisis parciales complejas. La TAC cerebral de control no evidenció cambios evolutivos (Figura 8).

Con el transcurso de los días, la paciente se recuperó de la afasia, manteniendo leve disartria y hemiparesia M4 braquiocrural izquierda, sin presentar otras complicaciones neurológicas. En cuanto al manejo, considerando la oclusión completa arterial y el riesgo de sangrado espontáneo gastrointestinal y de otros territorios, no se indicó tratamiento anticoagulante y se mantuvo con antiagregación plaquetaria cautelosa (5 días con $250 \mathrm{mg}$ /día de ácido acetilsalicílico y luego 100 $\mathrm{mg}$ /día). Por su buena evolución, se decidió alta con control en policlínico de fisiatría, neurología y cirugía vascular. Un mes después, equipo de neurólogos discutió el caso y decidieron mantener ácido acetilsalicílico y agregar propanolol (10 mg cada 12 h). Cirujano vascular concordó con mantener antiagregación plaquetaria y beta bloqueo. Cinco meses post alta se realizó angioresonancia magnética que demostró reconstitución de la arteria carótida interna derecha, confirmando la recanalización arterial.

\section{Discusión}

Las disecciones arteriales cérvico cefálicas son responsables de $0,4 \%$ a $4 \%$ de todos los accidentes vasculares encefálicos que se presentan en la población general, pero causan 5-20\% de los accidentes vasculares encefálicos entre los jóvenes. Entre las causas de disección arterial cervical y accidente cerebro vascular isquémico en un paciente joven, deben considerarse enfermedades del tejido conectivo, particularmente el síndrome de EhlersDanlos de tipo vascular ${ }^{11}$.

El síndrome de Ehlers-Danlos tipo vascular es una enfermedad genética poco frecuente que tiene como resultado una fragilidad constitutiva de los órganos ricos en colágeno de tipo III como lo son las arterias, venas, intestinos, pulmones, piel, hígado y bazo, convirtiéndola en una enfermedad de pronóstico grave debido a la tendencia impredecible de rupturas arteriales y de órganos ${ }^{12}$.
No está descrita su prevalencia ni incidencia en la literatura chilena y sólo se dispone de estudios realizados principalmente en Europa y Estados Unidos de Norteamérica.

El diagnóstico se basa en la clínica, las imágenes y la identificación de una mutación del gen COL3A1; sin embargo, los estudios genéticomoleculares confirmatorios son caros y sólo se hacen en Estados Unidos de Norteamérica. La utilización de técnicas no invasivas (ecografía Doppler, angioscanner, angioresonancia magnética) son absolutamente necesarias para el diagnóstico de disecciones arteriales o aneurismas en los pacientes con diagnóstico de síndrome de EhlersDanlos tipo vascular confirmado ${ }^{13}$. La arteriografía está contraindicada por el alto riesgo de rotura de la pared arterial, sin embargo, se reserva para los casos en que esté planeada una embolización ${ }^{14}$.

No existe un tratamiento curativo de esta enfermedad. El manejo debe ser conservador y preventivo. Farmacológicamente, se describe el uso de betabloqueadores como tratamiento preventivo de disección arterial, evitando la hipertensión arterial. El valor a largo plazo del tratamiento con celiprolol para prevenir las complicaciones vasculares es actualmente objeto de un ensayo clínico controlado, encontrándose este fármaco en proceso de validación (fase IV $)^{15}$.

No se ha demostrado beneficio con la administración de colágeno. Se debe evitar el uso de aspirina, clopidogrel y anticoagulantes por el riesgo de sangrado. En el caso presentado, la paciente fue manejada exitosamente sólo con antiagregación plaquetaria a bajas dosis. Sin embargo, se debe considerar que en el caso de una trombosis que se presente luego de una disección vertebral o carotidea, se debe plantear el uso de anticoagulación. Faltan estudios que demuestren la superioridad de la anticoagulación respecto a la antiagregación en cuanto a morbimortalidad ${ }^{16}$. El 4\% de los accidentes cerebrovasculares isquémicos secundarios a disección carotidea presentan hemorragia intracraneana y la mitad de estos casos son por ruptura de un aneurisma intracraneano previo ${ }^{17}$.

Teniendo en cuenta la fragilidad vascular, la cirugía es el último recurso a utilizar, debido a la alta mortalidad que conlleva (19-44\%). Ante esta eventualidad, debe darse preferencia a intervenciones mínimamente invasivas. Probablemente, en el futuro, la terapia génica sea una solución.

En cuanto al diagnóstico diferencial del síndro- 
me de Ehlers-Dalos de tipo vascular, en la infancia, se deben considerar principalmente los trastornos de la coagulación y el síndrome de Silverman; en la edad adulta, otros tipos de SED, el síndrome de Marfan y el síndrome de Loeys-Dietz.

\section{Referencias}

1. Pope FM, Narcisi P, Nicholls AC, Germain DP, Pals G, Richards AJ. COL3A1 mutations cause variable clinical phenotypes including acrogeria and vascular rupture. $\mathrm{Br}$ J Dermatol 1996; 135: 163-81.

2. Germain DP. Ehlers-Danlos syndromes. Clinical, genetic and molecular aspects. Ann Dermatol Venereol 1995; 122: 187-204.

3. Oderich GS. Current concepts in the diagnosis and management of vascular Ehlers-Danlos syndrome. Perspect Vasc Surg Endovasc Ther 2006; 18 (3): 206.

4. Beighton P, De Paepe A, Steinmann B, Tsipouras P, Wenstrup RJ. Ehlers-Danlos syndromes: revised nosology, Villefranche, 1997. Am J Med Genet 1998, 77: 31-7.

5. Fernández NF, Schwartz RA. A "hyperextensive" review of Ehlers-Danlos syndrome. Ped Dermatol 2008; 82: 242.

6. Pepin M, Schwarze U, Superti-Furga A, Byers PH. Clinical and genetic features of Ehlers-Danlos syndrome type IV, the vascular type. N Engl J Med 2000; 342: 673-80.

7. Byers PH: Ehlers-Danlos syndrome type IV: a genetic disorder in many guises. J Invest Dermatol 1995; 105:
311-3.

8. Emanuel BS, Cannizzaro LA, Seyer JM, Myers JC. Human a1 (III) and a $2(\mathrm{~V})$ procollagen genes are located on the long arm of chromosome 2. Proceedings of the National Academy of Sciences USA 1985; 82: 3385-9.

9. Germain DP, Herrera-Guzmán Y. Vascular EhlersDanlos syndrome. Ann Genet 2004; 47: 1-9.

10. Bergqvist D. Ehlers-Danlos type IV syndrome. A review from a vascular surgical point of view. Eur J Surg 1996; 162: 163-70.

11. Majersik JJ, Skalabrin EJ. Single-gene stroke disorders. Semin Neurol 2006; 26: 33-48.

12. Pope FM, Martin GR, Lichtenstein JR, Penttinen R, Gerson B, Rowe DW, et al. Patients with Ehlers-Danlos syndrome type IV lack type III collagen. Proc Natl Acad Sci USA 1975; 72: 1314-6.

13. Germain DP. The vascular Ehlers-Danlos syndrome. Curr Treat Options Cardiovasc Med 2006; 8: 121-7.

14. Slingenberg EJ. Complications during intravascular diagnostic manipulations in the Ehlers-Danlos syndrome. Neth J Surg 1980; 32: 56-8.

15. Boutouyrie P, Germain DP, Fiessinger JN, Laloux B, Perdu J, Laurent S. Increased carotid wall stress in vascular Ehlers-Danlos syndrome. Circulation 2004; 109: 1530-5.

16. Redekop GJ. Extracranial carotid and vertebral artery dissection: A review. Can J Neurol Sci 2008; 35: 146-52.

17. Schievink WI. Cerebrovascular Involvement in EhlersDanlos Syndrome. Curr Treat Options Cardiovasc Med 2004; 6: 231-6. 